\title{
A methodology for the optimisation of a mm-wave scanner
}

DOI:

$10.1117 / 12.2242118$

\section{Document Version}

Accepted author manuscript

Link to publication record in Manchester Research Explorer

\section{Citation for published version (APA):}

Stec, L., Podd, F., \& Peyton, A. (2016). A methodology for the optimisation of a mm-wave scanner. In Millimetre Wave and Terahertz Sensors and Technology IX: Phenomenology, Signatures and Modelling I (Vol. 9993). (Proceedings of SPIE). SPIE. https://doi.org/10.1117/12.2242118

\section{Published in:}

Millimetre Wave and Terahertz Sensors and Technology IX

\section{Citing this paper}

Please note that where the full-text provided on Manchester Research Explorer is the Author Accepted Manuscript or Proof version this may differ from the final Published version. If citing, it is advised that you check and use the publisher's definitive version.

\section{General rights}

Copyright and moral rights for the publications made accessible in the Research Explorer are retained by the authors and/or other copyright owners and it is a condition of accessing publications that users recognise and abide by the legal requirements associated with these rights.

\section{Takedown policy}

If you believe that this document breaches copyright please refer to the University of Manchester's Takedown Procedures [http://man.ac.uk/04Y6Bo] or contact uml.scholarlycommunications@manchester.ac.uk providing relevant details, so we can investigate your claim.

\section{OPEN ACCESS}




\title{
A methodology for the optimisation of a mm-wave scanner
}

\author{
L. Zoë Stec*, Frank J.W. Podd, Anthony J. Peyton \\ School of Electrical and Electronic Engineering, University of Manchester, Manchester M13 9PL \\ United Kingdom
}

\begin{abstract}
The need to detect non-metallic items under clothes to prevent terrorism at transport hubs is becoming vital. Millimetre wave technology is able to penetrate clothing, yet able to interact with objects concealed underneath.

This paper considers active illumination using multiple transmitter and receiver antennas. The positioning of these antennas must achieve full body coverage, whilst minimising the number of antenna elements and the number of required measurements. It sets out a rapid simulation methodology, based on the Kirchhoff equations, to explore different scenarios for scanner architecture optimisation.

The paper assumes that the electromagnetic waves used are at lower frequencies (say, 10-30 GHz) where the body temperature does not need to be considered. This range allows better penetration of clothing than higher frequencies, yet still provides adequate resolution.

Since passengers vary greatly in shape and size, the system needs to be able to work well with a range of body morphologies. Thus we have used two very differently shaped avatars to test the portal simulations. This simulation tool allows many different avatars to be generated quickly.

Findings from these simulations indicated that the dimensions of the avatar did indeed have an effect on the pattern of illumination, and that the data for each antenna pair can easily be combined to compare different antenna geometries for a given portal architecture, resulting in useful insights into antenna placement. The data generated could be analysed both quantitatively and qualitatively, at various levels of scale.
\end{abstract}

Keywords: Personnel screening, Walk through, Portal simulation, Kirchhoff, Millimetre wave, Security, Active, Scanner

\section{INTRODUCTION}

Airports, airline travel, and other transport hubs represent high profile targets to terrorist groups, with several attacks in 2016, such as the suicide bombings at Brussels Airport and Maalbeek metro station. These underline the ongoing need for robust and efficient personnel scanners at transport hubs for the detection of non-metallic terrorist devices concealed under clothing, as part of the measures to address this threat. Millimetre wave technology can help because the frequency of the electromagnetic waves is low enough to prevent the requirement of divesting outer clothing whilst being high enough to image and interact with threat objects beneath. Millimeter Wave Scanners can be either active or passive with several commercial examples of these in operation ${ }^{1,2,3,4}$. For checkpoint applications, active scanners are typically used as commercial 'Stand and Scan' models ${ }^{2}$ in use at airports around the globe ${ }^{5,6,7,8}$. Stand and Scan systems require the passenger to stop and pose whilst the scan takes place. Both IATA and the European Commission have issued statements to say continuous movement through a portal is a priority in airport security ${ }^{9,10}$. Therefore "walk through" portals offer a potentially faster throughput and thus a more effective screening and it is expected that such systems will emerge in the near future.

There are many papers published looking at using the millimetre and even sub-millimetre range for imaging, with 35 $\mathrm{GHz}^{11,12}, 94 \mathrm{GHz}^{13,14}$, and $500 \mathrm{GHz}^{15,16}$ as popular wavelengths. These wavelengths have good resolution, since they are much shorter than the dimensions of the body/object details to be examined. However, the higher frequencies can have difficulty penetrating thick clothing ${ }^{15,17}$. In addition, these shorter wavelengths/higher frequencies make the material appear rougher, resulting in more scattering and less power at the receiving antenna ${ }^{18}$. The shorter the wavelength, the lower the reflectivity becomes. For example, at $750 \mathrm{GHz}$, the reflectivity is only $3 \%$, while at $100 \mathrm{GHz}$ it is $14 \%{ }^{15}$. The use of EM waves in the 10-30 GHz range allows better penetration of clothing (especially if damp) compared with 
operation above $80 \mathrm{GHz}$, while still potentially providing adequate resolution. The recent availability of low-cost integrated circuits designed for the communication sector means this option brings the cost down ${ }^{17}$. In one study ${ }^{19}$,frequencies in the range $15-30 \mathrm{GHz}$ range are used to monitor people on a conveyor. At this range, body temperature does not need to be considered, unlike for waves in the $\mathrm{THz}$ range.

For the dynamic imaging requirement of the walk through configuration, the coverage of the EM radiation, and hence the coverage of the overall inspection may not be as well controlled as the "stand and scan" configuration. To address this issue, this paper sets out a simulation methodology to explore radiation body coverage, and consequently investigate different scenarios to compare walk through portal architecture, and facilitate the optimisation of antenna positioning.

There are many papers published using experimental results, with some ${ }^{20,21,22}$ describing the development of prototypes. However, experimental papers tend to describe specific conditions, and for the complex exploratory exercise, it would be too expensive and time-consuming to use experiments in the first instance. Simulation is a useful tool in this scenario. Many simulation systems use real data (e.g. ${ }^{16,23,24}$ have all used data generated by the development of QinetiQ's SPO series). Testing the most promising configurations with experimental data would help to verify the usefulness of the method presented here.

There are various methods that have been used to simulate millimetre wave exposure. For instance, ${ }^{19}$ used a Fourierbased imaging technique, explained $\mathrm{in}^{25}$, whilst ${ }^{26}$ used genetic algorithms to optimise antenna arrays by rotational scanning. A popular method ${ }^{12,27,28}$ is to adapt visible light ray tracing packages to millimetre wave conditions using physical optics. These ray-tracking methods require breaking down bodies and objects into a mesh, made up of triangular elements, with each element being examined using bidirectional reflectance distribution functions (BDRF).

An optimal imaging system would allow high passenger throughput, whilst keeping full body coverage. There are many high level aspects of imaging portal design that need to be considered to help the realisation of its design. There are a number of different permutations that should be considered, such as portal geometry and the effect of the public's physical variations. For instance, passengers going through security vary greatly in shape and size; it is important to look at how this affects the optimum portal geometry. The approach reported in the paper allows a very wide variety of body shapes to be examined. Two avatar examples, a male and a female, have been selected for particular consideration, which are very different in weight and height (the woman's height is at the 0.4th centile and the man's at the 98th centile). The advantage of using a simulation tool is that many different body shapes can be generated quickly, while finding large numbers of real volunteers of varying proportions is more difficult. Arm position is also an important parameter to consider; in these example simulations, the avatars both walked through with raised arms, for the sake of comparison. Other parameters such a walking gait can also be easily varied, although a single gait was used in this paper. The shape of the portal is very important as it determines the possible placement of the antennas and the proximity of the body to the antennas. The portal footprint will most likely need to be as small as possible, but the configuration should permit the public fast passage through, whilst achieving full radiation coverage.

This paper also considers the design of systems with coherent or incoherent active illumination. This usually requires multiple transmitter and receiver antennas. The positioning of these antennas within the portal geometry should allow full body coverage, whilst minimising the number of antennas and measurements. Full illumination of the passenger means that there is less chance of a dead zone where threat objects can be hidden. Minimising the number of measurements leads to a shorter measurement time and thus increased frame-rate and throughput. Finding an optimum number of antennas is important, since oversampling introduces unwanted noise to the system and can prove very expensive with the unnecessary antenna ${ }^{13}$, whilst undersampling could result in aliasing and reduced image sharpness. Furthermore, the simpler the configuration, the easier the system will be to calibrate ${ }^{26}$.

\section{BACKGROUND}

Ray tracing is an established technique for simulations of EM illumination applications, using the so called physical optics approach. For example, ${ }^{27}$ and ${ }^{28}$ used Open Fx and ${ }^{12}$ used Zemax to modify the ray tracing algorithm to be more suitable for millimetre wave and microwave simulations. The principle of ray tracing is that the ray is traced back to either the camera pixels or to the light source(s) (or in the case of millimeter wave imaging, the receiver to the transmitter). The number of rays per pixel can be increased for more accuracy. This process can continue without end, so recursive ray tracing is preferred, where the ray tracing stops at the appropriate maximum number of reflections. 
The Ray-tracing approach provides a fast approximation to the object surface's illumination levels and the object's surface sensitivity to transmitter and receiver antenna positions. The ray-tracing algorithm is very fast and can take just seconds to complete. However, the ray-tracing approach assumes that the wavelengths present in the signal are much smaller than the object size or object curvature and thus ignores scattering effects. Conversely, a simulation using the full Maxwell equations could more accurately predict the experimental results, but a typical implementation, using a Finite Difference Time Domain response for instance, can take many hours or days to produce a single result. This is due to the difference in scale; the dimensions of the objects being modelled and the portal geometry are many times larger than the small wavelength being used, so it is a numerically intensive method to investigate the problem of antenna positioning. A physical optics approach is more practical.

Kirchhoff diffraction theory approximates the scattering by a surface from a point source of radiation to a point receiver. By integrating the reflection, both amplitude and phase, over the surface should produce more accurate results than using the ray-tracing approximation, whilst still being fast enough to investigate the antenna positioning problem. Frequency dependent scattering can therefore be incorporated. This type of scattering occurs when the object dimensions are close to the wavelength of the ray, and describes the effect of the constructive and destructive interference of waves coming from different parts of the surface. This is likely to be relevant; for example, ${ }^{12}$ used a wavelength of $9 \mathrm{~mm}(35 \mathrm{GHz})$ and found that fingers and facial features were hard to discern.

The standard Kirchhoff approximation does not however account for the polarisation of the wave. This is important for accurate estimation of the reflection coefficient; the polarisation state will affect how it is scattered. Yet apart from ${ }^{9}$ it is usually not considered, since little work has been done on quantification so far. The effect of wave transmission into an object is also ignored, i.e. refraction and reflection. The latter is important when modelling the semi-transparency of skin in visible light ${ }^{30,31}$, but at millimeter wavelengths, penetration is shallow, at around $1 \mathrm{~mm}^{32}$.

The advantage of using Kirchhoff diffraction theory as the basis of the simulation, rather than standard ray tracing methods (various BDRFs and frequency independent) is that it can be used both for coverage and for reconstructing the signals, including scattering effects of objects.

\section{APPROXIMATION METHOD}

The implementation in this paper uses the Kirchhoff diffraction theory and accounts for the geometric energy spreading loss from the transmitter to the surface and from the surface to the receiver; and includes the angular dependent scattering coefficient of the surface.

The angle dependent factors are called "obliquity factors", or "inclination factors". The equations can be derived from the situation of a point source emitting waves that pass through a hole in a screen, and using Green's theorem. Different solutions result from different from boundary conditions. In our situation the same equations are employed, but rather than derived with holes, mesh facets are used.

For the point source emitter and detector, we have $\mathrm{e}^{33}$

$$
U\left(P_{R x}\right)=-\frac{i k\left|U\left(P_{T x}\right)\right|}{2 \pi} \oint_{S} \frac{e^{i k\left(R_{T x}+R_{R x}\right)}}{R_{T x} R_{R x}} \beta . d S
$$

where:

$\mathrm{R}_{\mathrm{Tx}}=$ distance from that point on the element to the transmitter.

$\mathrm{R}_{\mathrm{Rx}}=$ distance from that point on the element to the receiver.

$\mathrm{i}=$ imaginary number.

$\mathrm{k}=$ wavenumber.

$\mathrm{S}=$ the surface of the facet.

The inclination factor $(\beta)$ depends on the derivation:

$$
\beta=\left\{\begin{array}{c}
\frac{1}{2}\left(\cos \left(\theta_{T x, n}\right)-\cos \left(\theta_{R x, n}\right)\right), \text { Fresnel }- \text { Kirchhoff } \\
\cos \left(\theta_{T x, n}\right), \text { First Rayleigh }- \text { Sommerfeld } \\
-\cos \left(\theta_{R x, n}\right), \text { Second Rayleigh }- \text { Sommerfeld }
\end{array}\right\}
$$


Where:

$\Theta_{\mathrm{Tx}, \mathrm{n}}=$ the angle between the ray vector from the transmitter to the centre of the point on the surface and the surface normal at that position.

$\Theta_{\mathrm{Rx}, \mathrm{n}}=$ the angle between the ray vector from the receiver to the centre of the point on the surface and the surface normal at that position. A slight change to the equation is incorporated into this implementation; if the incoming or outgoing ray is beyond 90 degrees, then there is no scattering in this implementation.

The required mesh resolution is dependent on signal wavelength and ray distances. The closer the points on the surface are, the bigger the allowable distance from the element to the receiving antenna is. The distance between point $\mathrm{x}$ and any other point must be much less than the length scale, L, which is determined by the amount the medium varies over distance and is given by ${ }^{34}$

$$
L=\left(\frac{1}{k} \frac{d k}{d x}\right)^{-1}
$$

This paper reports only on the amplitude integration to estimate the coverage sensitivity of the object's surface to antenna positioning rather than integrating the amplitude and phase to reconstruct the received time-domain signal.

$$
\text { Element Sensitivity }=\sum_{\text {All Tx \& Rx }} \frac{1}{R_{T x} R_{R x}}\left(\cos \left(\theta_{T x, n}\right)-\cos \left(\theta_{R x, n}\right)\right)
$$

The following approximations are used in the derivation:

- The distance from the source to the surface, and from the surface to receiver are much larger than one sixth of the longest wavelength. This approximation is reasonable as long as the person is not too close to the antennas (say $>10 \mathrm{~cm}$ away), and the lowest frequency used is above say $6 \mathrm{GHz}$. This is for far field problems.

- The algorithm does not check if an element is behind another for that ray direction. Therefore we are not currently modelling shadow effects.

- It assumes all surfaces are $100 \%$ reflective, so clothing is consequently not modelled.

- Polarisation effects are not included. However, the polarisation, angle of incidence, and partial coherence of the illumination can have a significant effect on the scene appearance ${ }^{29}$.

As with the visible light ray tracing approaches discussed above, a surface mesh is used to describe the objects, but in this case, a Kirchhoff surface interaction model is assumed. The mesh implementation also assumes that if we can split the surface into small enough elements we can take the value at the centre of each element and multiply the result by the area of the element. The approach is particularly applicable to non-imaging systems, such as those based on object resonance, or harmonic generation. For imaging systems, the phase of the collected waves must also be integrated and a much higher density of rays per surface element is required.

\section{METHODS}

The purpose of this research is to provide a rapid and flexible simulation method to investigate different portal configurations, antenna positions, and excitation patterns. This paper demonstrates this simulation method by comparing different antenna geometries for a single portal architecture, with up to 18 antenna positions.

As mentioned in the introduction, efficient design of an active mm-wave portal requires various factors to be taken into account, which will affect both the sensitivity of the system for illicit item detection, and the cost and speed of the system (both passenger transit time and computational time). The parameters considered in this paper include pose, body morphology, portal configuration, and the distribution of antennas. The simulation is very fast, so these parameters can be altered and examined in detail. Nevertheless, to elucidate the principle, they are constrained here as described below:

\subsection{Pose and stride}

A person's pose can affect the coverage, particularly the way the arms are held. Stride length may also have an effect. For this initial trial, a single pose (arms raised, upper arm at $90^{\circ}$ to the body) was selected. Only one type of stride for each avatar was used.

\subsection{Body morphology}

An effective scanner design has to be flexible enough to accommodate many different sizes and shapes of body. Figure 1 shows the two body types used in the current investigation. The female is $1.42 \mathrm{~m}$ with a high BMI and the male is $1.95 \mathrm{~m}$ 
with a very low BMI. For ease of identification, they will be referred to as 'May' and 'John' respectively. Using MakeHuman $^{35}$, many more types of body morphology can be generated rapidly.

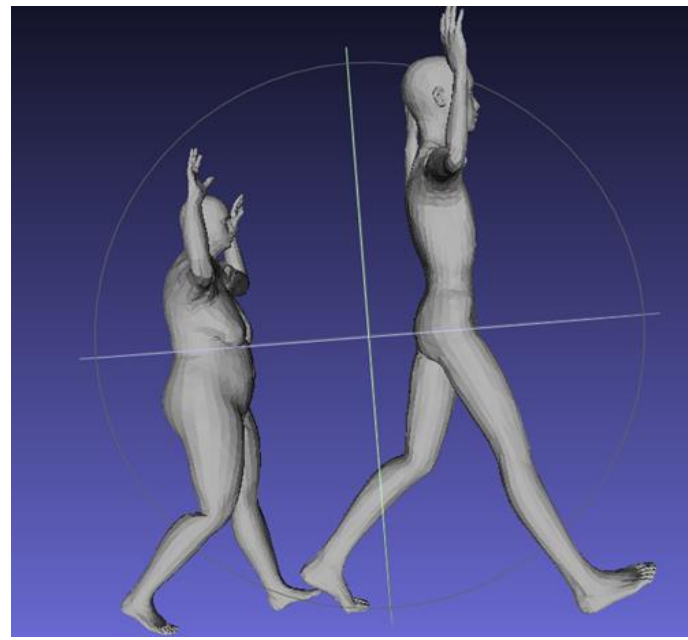

Figure 1. A comparison of the two avatars used, referred to as May and John

\subsection{Portal Configuration}

Three possible portal designs were considered, pictured in Figure 2 below. It was assumed that the two sides were not in contact with each other and consisted of three walls per side. We selected the 'Encircling' configuration for this report, as it offers much more opportunities for cross-panel measurements. Furthermore, the effect of varying distances and angles from the moving figure can be investigated.

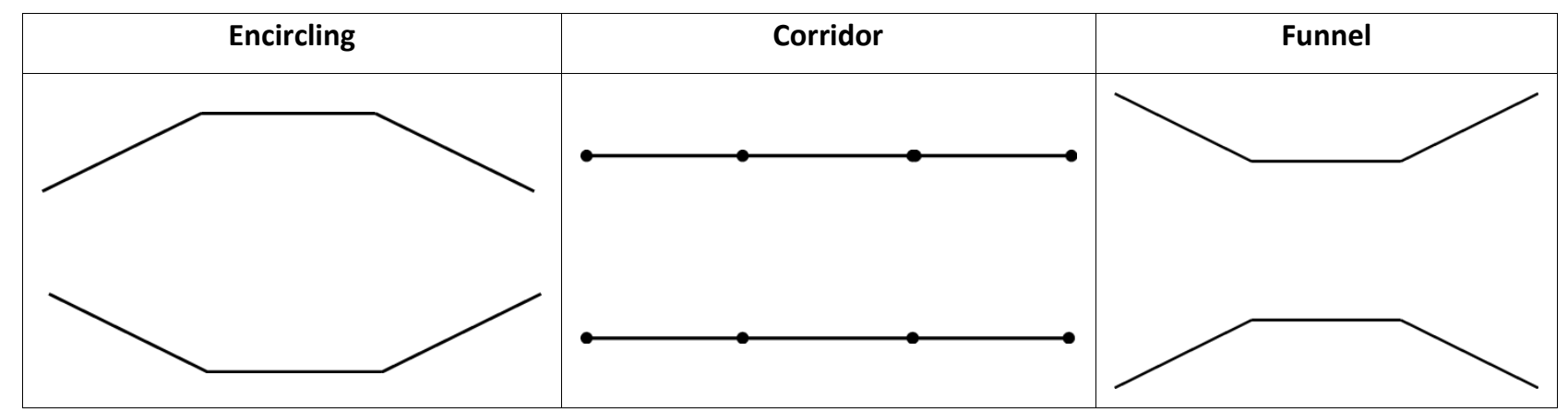

Figure 2. Three possible portal configurations two sides, three walls per side.

The specifications for the 'Encircling' geometry design were that the entrance and exit are $90 \mathrm{~cm}$ wide, as for current walk through scanners, and the angle of the first and last parts of the wall were at a $30^{\circ}$ angle with respect to the middle one, which is parallel to the direction of movement. Figure 3 shows an opened out view of the two sides. In this investigation it was decided to split each side into nine equally sized panels which will contain the antennas. Each panel is a square of $0.666 \mathrm{~m}^{2}$. They are stacked three panels high per wall, taking the total height to $2 \mathrm{~m}$, with three walls per side. However, the walking distance is only $1.82 \mathrm{~m}$, since the first and last walls are set at an angle. As the person enters, the right hand side wall with column $a-d-g$ is on their right and the LHS wall with columns $j-m-p$ is on their left. 


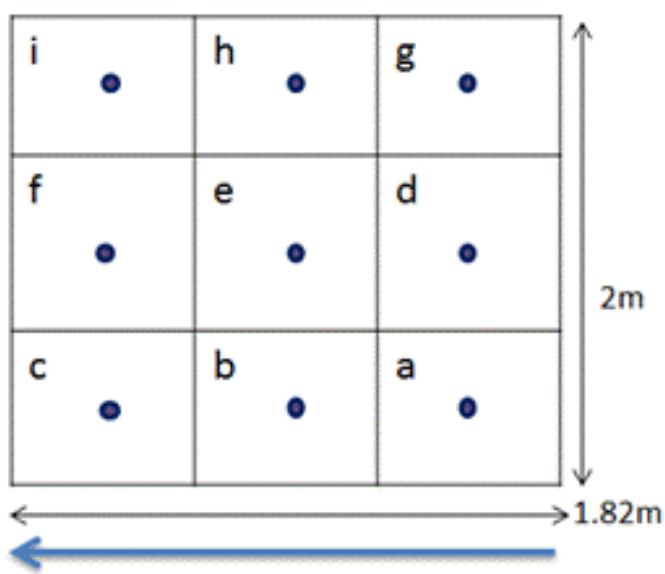

Walking direction

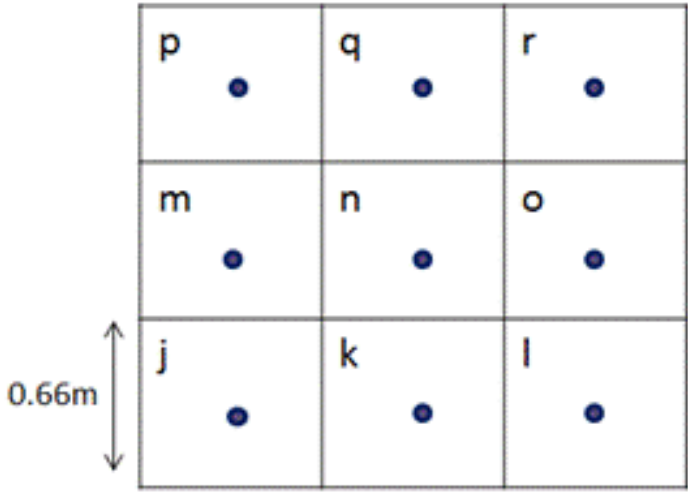

Walking direction

Figure 3. Plan showing the portal side dimensions and the orientation of the panels $a$ to $r$.

\subsection{Distribution of Antennas}

It is important to determine a suitable number and density of antennas in the portal, to help with the design architecture. We need to ask questions such as:

1. How can we find the smallest number of Rx and Tx that provides enough detail to build up a surface profile?

2. What is the effect of their distribution?

To help answer these questions, simulations were carried out, comparing differences between the results for a single antenna each in panels ' $a, e, i$ ' with three antennas within panel ' $a$ '. The configuration of the three panel sensor positions is illustrated below in Figure 5 below. Positions $a 1$ and $a 3$ are in the centre of the bottom left and top right quarters of sub-panel ' $a$ ', with $a 2$ being the default position, at the centre of the sub-panel. The vertical and horizontal distances between adjacent antennas within a panel are $0.165 \mathrm{~m}$, compared to $0.666 \mathrm{~m}$ between panels, i.e. $25 \%$ of the distance.

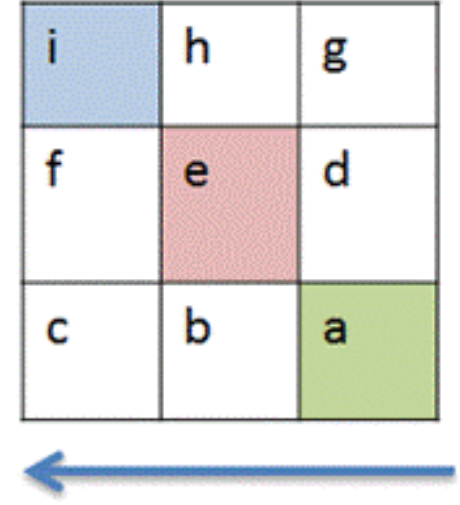

Figure 4 RHS Panel Configuration

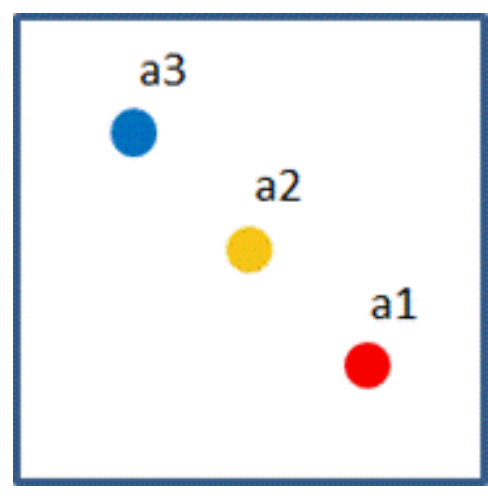

Figure 5 : Sub-panel Configuration for panel $a$

\subsection{Software Implementation}

The Human avatars were created using the MakeHuman software ${ }^{35}$. This is an Open Source program that starts with a basic human shape, and by using sliders and information boxes, a customised model can be created. Qualities such as 
age, height, race, sex, BMI and more detailed shapes (e.g. widening shoulders, increasing leg proportions) can be specified within minutes. The resulting avatars were saved as a Collada file (.dae) and imported into Blender.

Blender ${ }^{36}$ is open source software; this was used to animate the avatars, to enable them to walk the right distance past the coordinates corresponding to the sensors. It was also used to simplify the body meshes from 30,984 faces to 7,524, which shortened the simulation times from 3 hours to 30 minutes. Blender was also used to check the geometry and location of the sensors embedded in the portal. The frames making up the walk were exported in .stl format and a small selection was checked in Meshlab ${ }^{37}$ for number of faces and location of frames. All the frames were then processed in a Matlab ${ }^{38}$ program, designed to simulate the pattern of reflection using a pair of antenna either in a monostatic or bistatic configuration. The simulation used an adaptation of the Kirchhoff model and ray-tracing. As a simplification, the point source and a point detector scaled to a larger detector of $1 \mathrm{~m}^{2}$ in the program. The wavelength is not specified as a high frequency approximation is used for the coverage experiments. The last step was to use the output from the Matlab program to generate animations using Python ${ }^{39}$.

Five such animations for each set of sensor configurations were generated. Four of the animations showed the changing exposure as the avatar progressed through the portal at different views, namely front, back and both sides. The fifth animation showed the total exposure (for the full length of the portal) for each triangular face, by rotating the avatar.

A range of poses, body types and gaits can be implemented with this system. However, for illustration purposes, we have selected one pose and two body types as mentioned earlier.

\subsection{Processing of Data}

The output from the various simulations was a Matlab file containing data on the size and relative position of each triangular face, the location of the transmitters and receivers and the amplitude received at each triangular face. This last set of data can be converted into graph form to help to discover which faces are well-covered during the walk-through and which ones are mainly in shadow. For a qualitative view, a pictorial representation means that the effect of sensor location on body exposure is much easier to identify. This is provided by the animations/stills output from the Python program. Stills from the front, back and sides can show how the exposure varies over time. The rotating animation shows the total exposure throughout the passage of the portal.

A brief check of how the triangular faces were numbered in the mesh was carried out. Both avatars have 7,524 elements or faces in their mesh. Triangle numbering was not consistent between avatars, for instance element 100 is on different parts of the neck on each subject. However, the numbering for each body remained consistent throughout the walk. The total surface area for May is $1.53 \mathrm{~m}^{2}$, and $1.91 \mathrm{~m}^{2}$ for John. The mean element area is $2.04 \times 10^{-4} \mathrm{~m}^{2}$ and $2.54 \times 10^{-4} \mathrm{~m}^{2}$ respectively.

\section{RESULTS}

All the simulations were carried out with the arms out to shoulder height, raised forearms and palms facing forward. This pose was chosen as it is typical of current Stand and Scan systems. As expected, the results show that this pose provides high body coverage.

\subsection{Effect of body type on Coverage}

The two avatars differ in extremes of both height and BMI. The results show a clear difference in the distribution and coverage of the radiation. The total exposure for John is lower than for May, due to his longer strides give him a faster transition through the scanner, thus there are fewer data-acquisition frames (May takes 60 frames and John takes 48 frames to walk through the portal). However, this does not affect the patterns of coverage. Figure 6 shows one view of a 360 degree total for all the frames as the avatars walk through the portal. The transmitter and receiver are in the ' $a$ ' position, i.e. the lowest position on the right hand side as you enter the portal. The false colour spectrum scale is based on the percentage of EM radiation received, with a red hue being high and blue being low. Blue could mean no radiation at all or comparatively small amounts of EM radiation exposure for a short period of time, i.e. the false colour scale is relative. Their difference in height can also give a different coverage pattern - John is $1.92 \mathrm{~m}$ high compared to the top light height at $1.67 \mathrm{~m}$. 


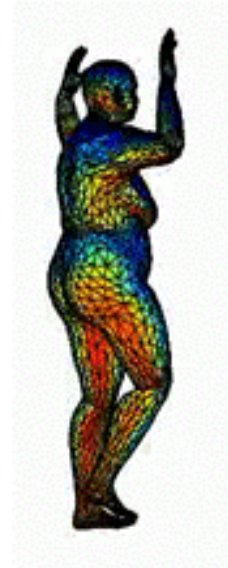

May aa

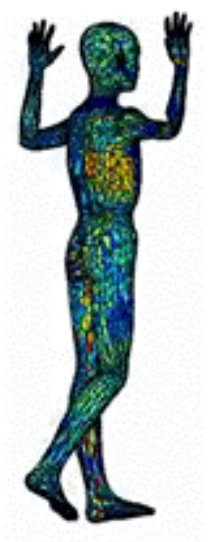

John $a a$

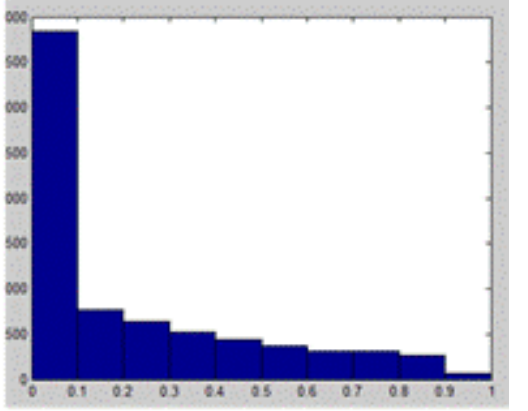

May $a a$

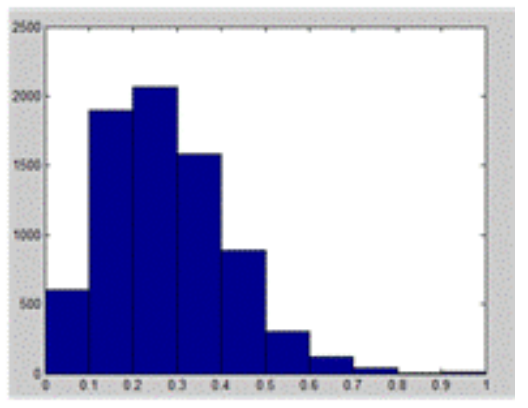

John $a a$

Figure 6 False colour map of amount of radiation received and histograms of proportion of radiation received against the number of elements in the avatar.

For the monostatic pair $a-a$, it seems that the May avatar has very strong coverage on her head, upper body, bottom and legs compared to John. As well as the difference in height and weight, the patterns on the legs will differ between the two, since they have different strides and therefore walking phases, compared to the antenna positions. The coverage is more even for John, whilst May shows areas of negligible illumination.

The histogram representations of the same data demonstrates that $51 \%$ of May's elements receive less than $10 \%$ of the energy emitted from the transmitter, i.e. 0.1 or less on the $\mathrm{x}$ axis. The $10 \%$ bin is assumed here as a measure of invalid results due to noise, although calibration might reveal a different figure in a realistic situation. Only $8 \%$ of John's elements are in this lowest category, with the majority of faces receiving 10-40\% of the energy from the transmitter. As expected from looking at the false colours on the avatars, May has more elements than John in the highest three categories- around $8.6 \%$ for the $80-100 \%$ category, whilst John has $0.7 \%$ for the equivalent categories.

Findings from these simulations indicated that the dimensions of the avatar do indeed have an effect on the pattern of illumination, and that the data for each antenna pair can easily be combined to compare different antenna geometries for a given portal architecture, resulting in useful insights into antenna placement.

\subsection{Monostatic versus bistatic pairs}

The two distributions shown in Figure 6 above (May $a$ - $a$ and John $a-a$ ) are typical patterns:

1. May's monostatic pairs fit the May $a$ - $a$ histogram pattern, i.e. large high intensity patches, with many areas of low coverage.

2. John's monostatic and bistatic pairs and May's bistatic pairs tend to be like the John $a-a$ distribution, i.e. the majority of faces receive some radiation, but few faces show high levels.

For example, if we compare the results for the monostatic pairs $a$ - $a$ and $r$ - $r$ with the bistatic pair $a-r$, as in Figure 7, we can see the two distinct patterns; $a-a$ on the bottom right at the entrance, and $r-r$ at the top left at the exit have bright spots on the back and on the front respectively. Only one side view is shown for each one, since the other has negligible coverage. 


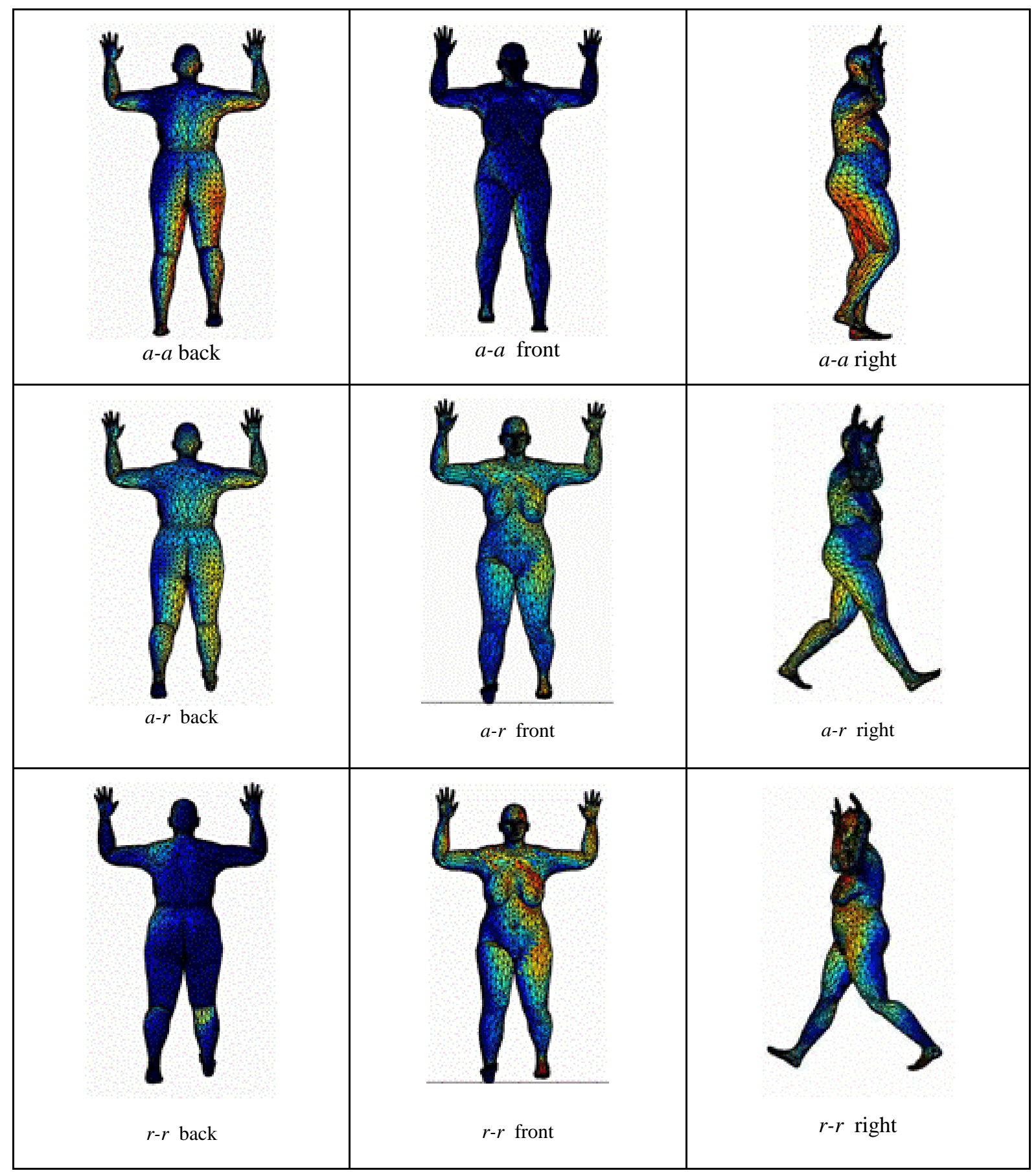

Figure 7 Comparison of electromagnetic exposure for May for monostatic pairs $a$ - $a$ and $r-r$ and bistatic pair $a-r$

On the other hand, the bistatic pair $a-r$ displays both bright spots in the same location as $a-a$ on the back and bright spots analogous to those for $r-r$ on the front, so much of the body is illuminated ( only the right side is shown in Figure 7). However, the bright spots are less intense than for the monostatic pairs and there are lower maximums for the bistatic pair. The difference in coverage can be seen by the data in Table 1 . 
Table 1 Comparative Exposure levels for May's monostatic pairs $a-a$ and $r-r$ and bistatic pair $a-r$

\begin{tabular}{|l|l|l|l|l|l|l|l|l|l|l|l|}
\hline $\begin{array}{l}\text { Received } \\
\text { radiation }\end{array}$ & $\mathbf{0 . 1}$ & $\mathbf{0 . 2}$ & $\mathbf{0 . 3}$ & $\mathbf{0 . 4}$ & $\mathbf{0 . 5}$ & $\mathbf{0 . 6}$ & $\mathbf{0 . 7}$ & $\mathbf{0 . 8}$ & $\mathbf{0 . 9}$ & $\mathbf{1 . 0}$ \\
\hline $\boldsymbol{a}-\boldsymbol{a}$ & $51 \%$ & $10 \%$ & $8 \%$ & $7 \%$ & $6 \%$ & $5 \%$ & $4 \%$ & $4 \%$ & $4 \%$ & $1 \%$ \\
\hline $\boldsymbol{a}-\boldsymbol{r}$ & $8 \%$ & $11 \%$ & $18 \%$ & $19 \%$ & $17 \%$ & $14 \%$ & $9 \%$ & $2 \%$ & $1 \%$ & $1 \%$ \\
\hline $\boldsymbol{r}-\boldsymbol{r}$ & $43 \%$ & $11 \%$ & $9 \%$ & $8 \%$ & $7 \%$ & $7 \%$ & $6 \%$ & $5 \%$ & $3 \%$ & $2 \%$ \\
\hline
\end{tabular}

For the bistatic antenna pair $a-r$, the proportion of elements receiving less than $10 \%$ of the radiation emitted is only around $8 \%$, compared to $51 \%$ and $42 \%$ for the monostatic pairs $a-a$ and $r$-r . Conversely, the percentage of elements on the last 3 bins is only $2.6 \%$ for $a-r$, compared to the monostatic pairs (both at $9 \%$ ). These figures also indicate evidence that the monostatic pair $r-r$ is slightly better situated for effective illumination than $a-a$.

The equivalent data for John shows that instead of 7 or $8 \%$ of the elements receiving less than $10 \%$ of the radiation for $a$ $r$, the bistatic pair reduces this figure to $0.4 \%$. In addition, $62 \%$ of elements receive between $41 \%$ and $60 \%$ of the emitted radiation for the bistatic configuration, compared to say $a$-a, where $73 \%$ of elements receive between $11 \%$ and $40 \%$ radiation. Again $r-r$ performs slightly better, $68 \%$ of the elements receive between $31 \%$ and $60 \%$ of the emitted radiation.

Comparison of individual monostatic pairs shows the effect of antenna positioning. For May for example, the upper exit panels $r-r$ and $i-i$ provide better illumination than the lower entrance panel $a-a$.

\subsection{Distribution of antennas}

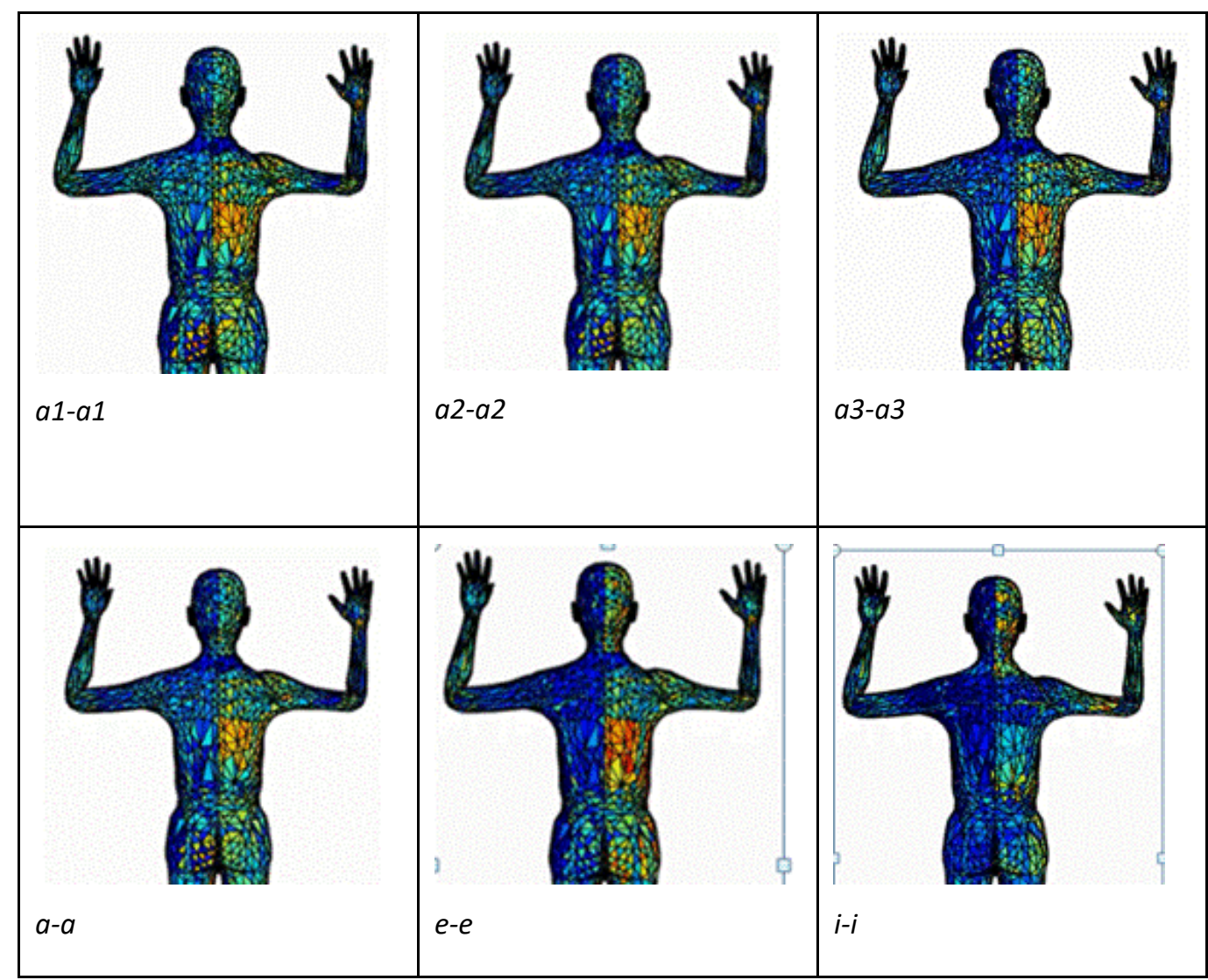

Figure 8: Comparison of difference in radiation patterns for 3 monostatic antenna in three separate panels $(a-e-i)$ and within one panel (a123)

Increasing the number of antennas would obviously result in the antennas becoming more closely spaced. We can look at the effect of having more antennas on radiation coverage by comparing three antennas within a panel in a diagonal 
configuration to three panels also in a diagonal configuration, as described in the methods section above. The data below combines the data for three monostatic pairs to see if there is an improvement in the illumination of the avatars. Figure 8 compares the coverage results of the three antenna within a panel (a1-a1, a2-a2,a3-a3) to that of three panels ( $a-a, e-e$, $i-i)$ for the John avatar. The images show how little quantitative difference there is between the antennas within a panel. On the other hand, there is a large amount of difference between $a$ - $a, e$-e and $i-i$, partly because $a$ - $a$ mainly illuminates the back and $i-i$ mainly illuminates the front of the bodies.

The difference is quantified in Table 2. The proportion of elements receiving $10 \%$ of the radiation or less for John is $8 \%$ for the monostatic pair $a-a$. This is similar for 3 antenna within a panel (a123) at $7.3 \%$. All the other categories for three antennas to a panel are similar to one antenna in the $a$ sub-panel. On the other hand, the below $10 \%$ figure for John $a-e-i$ is only $1.3 \%$, and the peak (meaning the bin with the highest proportion of elements with that level of illumination) has been pushed up by two bins/categories (40-50\% instead of 20-30\%). The figures for May show the same trends, with the below $10 \%$ bin containing around $17 \%$ fewer elements for May $a-e-i$ than the $a$ panel configuration.

Table 2: Distribution of the proportion of radiation reaching the receiver, comparing one monostatic pair (a-a) with a combination of three monostatic pairs in 3 different panels $(a-e-i)$ and within one panel (a123) for both avatars

\begin{tabular}{|l|l|l|l|l|l|l|}
\hline $\begin{array}{l}\text { Received } \\
\text { badiation } \\
\text { bins }\end{array}$ & $\begin{array}{l}\text { MAY } \\
a-e-i\end{array}$ & $\begin{array}{l}\text { MAY } \\
a 123\end{array}$ & $\begin{array}{l}\text { MAY } \\
\boldsymbol{a}-\boldsymbol{a}\end{array}$ & $\begin{array}{l}\text { JOHN } \\
a-e-i\end{array}$ & $\begin{array}{l}\text { JOHN } \\
a 123\end{array}$ & $\begin{array}{l}\text { JOHN } \\
\boldsymbol{a}-\boldsymbol{a}\end{array}$ \\
\hline 0.1 & $33 \%$ & $50 \%$ & $\mathbf{5 1 \%}$ & $1 \%$ & $7 \%$ & $\mathbf{8 \%}$ \\
\hline 0.2 & $14 \%$ & $11 \%$ & $\mathbf{1 0 \%}$ & $12 \%$ & $25 \%$ & $\mathbf{2 5 \%}$ \\
\hline 0.3 & $15 \%$ & $8 \%$ & $\mathbf{8 \%}$ & $21 \%$ & $27 \%$ & $\mathbf{2 7 \%}$ \\
\hline 0.4 & $13 \%$ & $7 \%$ & $\mathbf{7 \%}$ & $25 \%$ & $21 \%$ & $\mathbf{2 1 \%}$ \\
\hline 0.5 & $10 \%$ & $6 \%$ & $\mathbf{6 \%}$ & $22 \%$ & $12 \%$ & $\mathbf{1 2 \%}$ \\
\hline 0.6 & $7 \%$ & $5 \%$ & $\mathbf{5 \%}$ & $11 \%$ & $4 \%$ & $\mathbf{4 \%}$ \\
\hline 0.7 & $5 \%$ & $5 \%$ & $\mathbf{4 \%}$ & $5 \%$ & $2 \%$ & $\mathbf{2 \%}$ \\
\hline 0.8 & $2 \%$ & $5 \%$ & $\mathbf{4 \%}$ & $2 \%$ & $1 \%$ & $\mathbf{1 \%}$ \\
\hline 0.9 & $1 \%$ & $3 \%$ & $\mathbf{4 \%}$ & 0 & 0 & $\mathbf{0}$ \\
\hline 1 & 0 & $1 \%$ & $\mathbf{1 \%}$ & 0 & 0 & $\mathbf{0}$ \\
\hline
\end{tabular}

\subsection{Combined Totals}

Combining all May's results for the nine antenna on the right hand side (panels $a$ to $i$ ), the pattern looks very similar to combining the results for the $a, e$ and $i$ panels, regardless of whether it is just for the monostatic pairs or all the bistatic pairs are included. For example, if just the monostatic pairs are considered, the under $10 \%$ value for the elements is $32 \%$ of the elements, whilst if the bistatic pairs are included, the figure becomes $30 \%$. The peak occurs at $30 \%$ for both calculations. Meanwhile for John, the below $10 \%$ figure is very low indeed, at $0.8 \%$ of elements, and the element peak lies at $40 \%$.

The calculation for the total for all permutations on both sides of the portal was calculated for May as shown below. Figure 9 shows that only $4.5 \%$ of the elements had an exposure of $10 \%$ or below, and the peak occurred between $21-$ $49 \%$. 


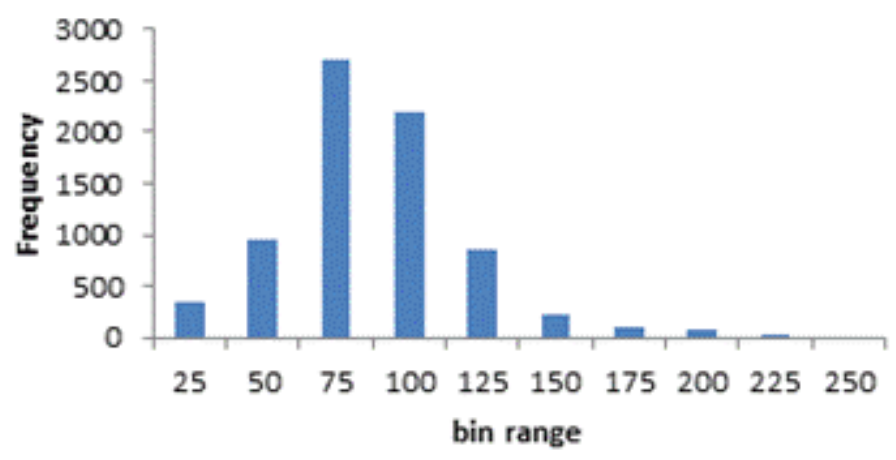

Figure 9: Total for all permutations on both sides of the portal for May

It is difficult to do this type of element by element sensitivity analysis using a full Maxwell simulation, even if computation time was not an issue.

\section{DISCUSSION AND CONCLUSIONS}

These techniques can be a very effective tool for comparing the effects of portal architecture on the levels of illumination. This simulation approach enables the comparison of data at different levels, the most detailed level being facet analysis, giving a fine grained appreciation of the sensitivity to the system geometry and following a single facet through a walk cycle. Having access to the element data means that a focus on specific areas of the body is possible, such as the areas where illicit items are most likely to be concealed.

At the panel level, the technique can show which panels are positioned to maximise radiation coverage and at a high level, it enables an overall visualisation, e.g. comparing the walkthrough frame by frame shows the changes throughout the walk through. This approach is valuable for looking at overall antenna geometry, such as examining the effect of placing the antennas into panels, how many panels to use, and where to locate them.

For each of the different levels of detail, this simulation approach facilitates:

- A comparison of how body shape and walking gait can affect the body surface coverage for given antenna configurations.

- Initial optimisation of the antenna density. The conditions for this simulation indicated that a tripling in antenna density had a very low effect on the facet sensitivity. For imaging systems, the number of antennas will also however be a factor in image reconstruction and imaging artefacts.

The approach does have caveats. It does not investigate sensitivity to the inverse problem, i.e. the actual image reconstruction, and it is an approximation rather than a full Maxwell solution. On the other hand, it has the advantage of permitting both qualitative and quantitative data analysis easily.

This paper shows an approach for optimising coverage. However, to perform a particular optimisation, the constraints on portal design and the number of antennas must be determined. Basing the simulation physics on Kirchhoff diffraction theory, rather than standard raytracing methods means that it can be used in a computationally fast implementation for coverage and non-imaging requirements. Whilst it could also be used for reconstructing the signals, including scattering effects of objects. However for the latter, the phase response and frequency bandwidth must also be included; and a higher ray density must be used, which increases the computational requirements.

\section{REFERENCES}

[1] QinetiQ, "DSEI first look: QinetiQ SPO-NX stand-off threat detection system" http://www.dsei.qinetiq.com/news/Pages/spo-nx.aspx (24 August 2016).

[2] L3, "L3 Security and Detection systems: Provision 2," http://www.sds.1-3com.com/advancedimaging/provision2.htm (20 July 2016).

[3] Teraview, "Terahertz Stand-off Explosives Detection System," http://www.teraview.com/products/terahertzpulsed-spectra-3000/terahertz-standoff-detection.html (24 August 2016). 
[4] Microsemi, "AllClear: Next Generation People Screening With Microsemi Millimeter Wave Technology," http://www.microsemi.com/index.php?option=com docman\&task=doc download\&gid=132519 (9 August 2016).

[5] Ashcroft, E., "Bristol Airport unveils "full body" security scanners," Bristol Post, 1 August 2016 http://www.bristolpost.co.uk/bristol-airport-unveils-full-body-security-scanners/story-29572471detail/story.html (2016).

[6] Allett, T., "Centralised Security," Airports International, March 2015, file:///C:/Users/Zoe/Downloads/Centralised_Security_Airports_International_March_2015.pdf (2015).

[7] Transport Security Administration, "Imaging Technology," 6 January 2010, http://www.tsa.gov/approach/tech/imaging_technology.shtm (2010).

[8] Manchester Airport, "Body Scanners at Manchester Airport," http://www.manchesterairport.co.uk/at-theairport/guides-to-travelling/security-and-fasttrack/body-scanners (2016).

[9] European Commission Directorate-General Enterprise \& Industry in collaboration with Decision Études \& Conseil, "Study on the Competitiveness of the EU security industry," p.24, November 2009, http://ec.europa.eu/dgs/home-affairs/elibrary/documents/policies/security/pdf/study_on_the_competitiveness_of_the_eu_security_industry_en.pdf (2009).

[10] IATA, "Checkpoint of the Future. Executive Summary," p.14, http://www.iata.org/whatwedo/security/Documents/cof-executive-summary.pdf (2012).

[11] Salmon, S.A., Appleby R., Price, S., "Scene simulation of passive millimetre wave images of plastic and metal objects," Proc. SPIE 4719, 397-401 (2002).

[12] Grafulla-González, B., Lebart, K., Harvey, A.R., "Physical optics modelling of millimetre-wave personnel scanners," Patt. Recog. Lett. 27, 1852-1862 (2006).

[13] Zhang, L., Hao, Y., Parini, C.G., Dupuy, J., “An Investigation of Antenna Element Spacing on the Quality of Millimeter Wave Imaging,” 2008 IEEE Ant. Prop. Soc. Int. Symp., 1-9, 4050-4053 (2008).

[14] Alefs, B.G., Hollander, R. J. M., Nennie, F. A., van der Houwen, E. H., Bruijn, M., van der Mark, W., Noordam, J. C., "Thorax biometrics from millimetre wave images," Patt. Recog. Lett. 31, 2357-2363 (2010).

[15] Sinclair, G.N., Appleby, R., Coward, P.R., Price, S., "Passive millimetre wave imaging in security scanning," Proc. SPIE 4032, 40-45 (2000).

[16] Salmon, S.A., "Scene simulation for passive and active millimetre and sub-millimetre wave imaging for security scanning and medical application", Proc. SPIE 5619, 129-135 (2004).

[17] Podd, F.J.W., David, M., Iqbal, G., Hussain, F., Morris, D., Osakue, E., Yeow, Y., Zahir, S., Armitage D.W., Peyton, A.J., "Impulse Radar Imaging system for concealed object detection," Proc. SPIE 8900, 890000 (2013).

[18] Federal Communications Commission, Office of Engineering and Technology, "Millimeter Wave Propagation: Spectrum Management Implications," 70, (1997).

[19] Gonzalez-Valdes, B., Alvarez, Y., Rodriguez-Vaquiero, Y., Arboleya-Arboleya, A., Garcia-Pino, A., Rappaport, C.M., Las-Heras, F., Martinez-Lorenzo, J.A., "Millimeter Wave Imaging Architecture for On- TheMove Whole Body Imaging," Trans.Ant and Prop., 64, (6), 2328-2338 (2016).

[20] Rozban, D., Aharon A., Abramovich A., Levanon, A., Yitzhaky, Y., Kopeika, N.S., "Detection of hidden objects using a real-time 3-D millimetre-wave imaging system," Proc. SPIE 9253, 7-13 (2014).

[21] Heinz, E., May, T., Born, D., Zieger, G., Anders, S., Zakosarenko, V., Meyer, H.-G., Schäffel, C., "Passive 350 GHz Video imaging Systems for Security Applications," J Infrared Milli Terahz Waves 36, 879-895 (2015).

[22] Anderton, R.N., Appleby R., Coward P.R., Kent P.J., Price, S., Sinclair, G.N., Wasley, M.R.M., “ Security Scanning at 35 GHz," Proc. SPIE 4373, 16-23 (2001).

[23] Haworth, C.D., Petillot, Y.R., Trucco, E., "Image processing techniques for metallic object detection with millimetre-wave images," Patt. Recog. Lett. 27, 1843-1851 (2006).

[24] Salmon, N.A. "Polarimetric scene simulation in millimetre wave radiometric imaging," Proc. SPIE 5410, 260269 (2004).

[25] Alvarez, Y., Rodriguez-Vaquiero, Y., Gonzalez-Valdes, B., Las-Heras, F., Garcia-Pino, A., "Fourier-Based Imaging for Subsampled Multistatic Arrays," IEE Transactions on Antennas and Propagation, 64 (6), $2557-$ 2562 (2016).

[26] Lucotte, B.M., Grafulla-González B., Harvey, A.R. “Array rotation aperture synthesis for short-range imaging at millimeter wavelengths,” Radio Science 44(1), RS1006 (2009). 
[27] Appleby, R., Petersson, H., Ferguson, S., “Concealed Object Stand-off Real-Time Imaging for Security: CONSORTIS,” Proc. SPIE 9462, 946204 (2015).

[28] Appleby, R. and Ferguson, S., "Phenomenology and system engineering of micro- and nano-antenna FPA sensors for detection of concealed weapons and improvised explosive devices," Proc. SPIE 8373, 29- 40 (2012).

[29] Qi, F., Ocket, I., Schreurs, D., Nauwelaers, B., "A system-level simulator for indoor mmW SAR imaging and its applications," Optics Express 20, 23811-23820 (2012).

[30] Li, L. and Ng, C.S., “A Physically-based Human Skin Reflection Model,” ICAI'09, 25-30 (2009).

[31] Weyrich, T., Wojciech, M., Pfister, H., Bickel, B., Donner, C., Tu, C., McAndless, J., Lee, J., Ngan, A., Jensen, H.W., Gross, M., “Analysis of Human Faces using a Measurement-Based Skin Reflectance Model,” ACM Trans. on Graphics, 25(3),1013-1024 (2006).

[32] Wu, T., Rappaport T.S, Collins, C.M., "The Human Body and Millimeter-Wave Wireless Communication systems: Interactions and Implications," ICC, 2423-2429 (2015).

[33] Goodman, J, [Introduction-to-Fourier-Optics] McGraw-Hill series in Electrical and Computer Engineering, Bogotá, p.51 (1988).

[34] Lucke, R.L., “Rayleigh-Sommerfeld Diffraction vs Fresnel-Kirchhoff, Fourier Propagation, and Poisson's Spot," Defense Technical Information Center, 30 December 2004 http://www.dtic.mil/dtic/tr/fulltext/u2/a429355.pdf (2004)

[35] http://www.makehuman.org/

[36] https://www.blender.org

[37] http://www.meshlabjs.net

[38] https://nl.mathworks.com/

[39] https://www.python.org/ 